\section{Werte. Warum es sie gibt, wenn wir sie brauchen}

\section{Dörte Gebhard}

Werte gelten den einen als überaus beständig, den anderen erscheinen Werte dem rasanten Wandel oder gar Verfall unterworfen. Beide Sichtweisen und ihre Mischformen verdienen Aufmerksamkeit.

Werte sind Überlebenskünstler durch Zeiten und Kulturen hindurch. Gerade ihre Flexibilität und die Weite der Interpretationen machen sie zu einer herausfordernden, kritischen Kommunikationshilfe für die Diakonie in pluralistisch geprägten Zeiten.

\section{Wert(er)findung? Eine Frage des Zugangs}

Werte und alles, was man ihnen an positiven Konsequenzen zutraut, sind aus vielen gegenwärtigen, gesellschaftlichen Gesprächskulturen nicht wegzudenken. Wertdiskussionen sind dabei so alt wie das Nachdenken über die Ökonomie, über das, was sich lohnt und bezahlt macht, über jenes auch, das seinen Preis, sein Angebot und seine Nachfrage hat, über alles, das einen Wert hat, haben könnte oder bekommen sollte. Dieser spezifische Verständnishorizont ist nicht ganz verloren gegangen, als die Werte in die Sphären der Philosophie und der Theologie, in ursprünglich durchaus wirtschaftsferne Lebensbereiche, vordrangen.

Wertdefinitionen sind so vielfältig wie die Listen von möglichen Werten lang sind. Sehr weit und mit leichten Varianten verbreitet hat sich die folgende, allgemeine Definition, die jedoch auch ihre Wurzeln in der Unternehmensberatungskultur hat:

«Werte (Wertvorstellungen) sind erstrebenswerte und subjektiv moralisch als gut befundene Eigenschaften, Qualitäten oder Glaubenssätze. Aus festgelegten und gewichteten Werten (Normen) resultieren Denkmuster, Handlungsmuster und Charaktereigenschaften. In Folge entstehen Ergebnisse (Resultate, Produkte, Erlebens-
Zustände), welche die gewünschten «werthaltigen` Eigenschaften besitzen bzw. vereinen.»1

Da Werte unter anderem das Gewand von Glaubenssätzen anziehen können, ist theologische Reflexion notwendig. Eberhard Jüngel erkennt den Diskurs über Werte als «durchaus hinderlich» ${ }^{2}$ für die Wahrheitsfrage, die sich stelle und im christlichen Glauben beantworten lasse. «Christliche Wahrheitserfahrung ist die radikale Infragestellung der Rede von Werten und des Denkens in Werten.» ${ }^{3}$ Aber auch diese Überzeugung befreit nicht von der Pflicht, sich mit den aktuell vorhandenen Wertdebatten kritisch auseinanderzusetzen.

Verhandelt wird an den Wertfragen häufig das Umfassende, das Grundsätzliche, das Fundamentale. Es geht dann um nichts Geringeres als z. B. um die Werte Europas ${ }^{4}$ oder der Schweiz, ${ }^{5}$ die Werte der einen oder die Werte der ganz anderen Partei, häufig um christliche Werte, Werte in, für und mit Familien oder solche Werte, die eine Kindertages-

1 URL: www.wertesysteme.de, abgerufen am 23. 2. 2018; ein Projekt der Unternehmensberatung Da Vinci 3000. The Academy for Business and Life, Köln.

2 Eberhard Jüngel, Wertlose Wahrheit. Christliche Wahrheitserfahrung im Streit gegen die 'Tyrannei der Werte, in: ders., Wertlose Wahrheit. Zur Identität und Relevanz des christlichen Glaubens. Theologische Erörterungen III, Tübingen 22003, 90-109 (90).

3 Ebd., 100.

4 Vgl. Heinrich August Winkler, Ohne Werte ist Europa nichts, in: ZEIT ONLINE vom 19. 11. 2017, abgerufen am 19. 2. 2018. Der Aufsatz beginnt: «Es war einmal eine Wertegemeinschaft, die sich Europäische Union nannte.»

5 Vgl. die Leserdebatte der NZZ, Was sind überhaupt Schweizer Werte?, vom 1. 8. 2017, abgerufen am 19. 2. 2018. Im Einführungstext heisst es: «Freiheit, Gleichheit und Solidarität seien christliche Werte, und der Islam gehöre nicht zur Schweiz, da er Land und Kultur nicht historisch geprägt habe, sagte etwa CVP-Präsident Gerhard Pfister vergangenes Jahr über die Schweizer Werte. Dieser Beschreibung konnten jedoch viele nichts abgewinnen.»
Jahrbuch Diakonie Schweiz 2 (2018) - ISSN 2504-3994

Dieser Text ist lizenziert unter einer Creative Commons Namensnennung 4.0 International Lizenz (CC BY 4.0): (https://creativecommons.org/licenses/by/4.0/).
Jahrbuch Diakonie Schweiz 2 (2018) http://dx.doi.org/10.22018/JDS.2018.5 
stätte, eine Künstlerkolonie oder eine (Unternehmens-)Kultur speziell prägen oder prägen sollen oder prägen sollen könnten.

Trotz mahnender Stimmen wie jener von Ulrich Körtner ${ }^{6}$ werden auch in der Diakonie entsprechende Wert(er)findungsprozesse initiiert, werden Werte gewählt und begründet und Leitbilder voller Werte formuliert. Eine grundlegende Ablehnung jeglicher Diskussionen über Werte empfiehlt sich nicht.

Denn vor allem im Alltag diakonischen Tuns, im Deutschkurs für Neuankömmlinge ebenso wie bei der Pflege Hochbetagter und Sterbender, begegnen die sehr unterschiedlichen und nicht ohne Weiteres miteinander vereinbaren Wertmilieus, Wertverständnisse, Wertkulturen von Menschen, so dass die Diakonik nicht von der - durchaus kritischen Teilnahme an gesellschaftlichen Wertdiskursen dispensiert werden kann.

Als hätte er die diakonischen Herausforderungen vor Augen, malt Jüngel ein farbenfrohes und kontrastreiches Bild dessen, was dadurch bevorsteht:

«Es kommt zum Wertkonflikt [...]. In ihr, im bunten Menschenleben, tritt der jeweilige Wert darüber hinaus nur gar zu gern mit einem rigorosen Anspruch auf, der andere Werte - die nicht einmal in Konflikt mit diesem Wert geraten müssen, sondern nur wie dieser die Situation mitbestimmen wollen - zu verdrängen sucht. Nicht das Sein der Werte, wohl aber die Realisierung der Werte führt leicht zum Rigorismus, ja Fanatismus im Blick auf einen bestimmten Wert.»7

6 Vgl. Ulrich H. J. Körtner, Diakonie und Öffentliche Theologie. Diakoniewissenschaftliche Studien, Göttingen 2017, 115: «Die Rückbesinnung auf (Werte), welche diakonische Einrichtungen für sich reklamieren, muss in diesem Zusammenhang durchaus kritisch gesehen werden. Zumindest für evangelische Ethik gibt es gute Gründe, sich zu jeder Form der Wertethik kritisch zu verhalten und auf die Gefahren einer 〈Tyrannei der Wertes hinzuweisen, verkündigt doch das Evangelium - mit Eberhard Jüngel gesprochen - eine (wertlose Wahrheit.»

7 Jüngel, Wahrheit (Anm. 2), 97.
Dieser Beitrag zeigt exemplarisch, was Auseinandersetzungen um Werte hervorbringen, so dass aktuelle Phänomene eingeordnet und differenziert - siehe da - bewertet werden können.

\section{Wertentdeckungen? Eine Frage des Verständnisses}

Immer wieder begegnet man bestimmten Sternbildern an vorgestellten Wertehimmeln, die für den menschlichen Betrachter scheinbar unverrückbar leuchten, gemäss den Wertastronomen wieder gesucht werden müssten, sich aber in Wirklichkeit in rasanter Bewegung befinden. Rekurriert wird in diesen Fällen auf die angenommene Kontinuität von Werten. Werte sollen garantieren, dass bleibt, was war, oder erreichen, dass wieder wird, was nicht mehr ist. Man möchte gelegentlich sogar unmittelbar «Mit Werten Wert schaffen», ${ }^{8}$ jedoch gemäss Selbstverständnis keineswegs neue Werte kreieren. An der gemeinnützigen Stiftung «Zukunft $\mathrm{CH}$ », die das zitierte Motto als Untertitel führt, wird hier exemplarisch gezeigt, mit welcher Vehemenz die allgemeine Wiederentdeckung - indes sehr ausgewählter - Werte verlangt wird.

«Eine Gesellschaft mit schwachen Werten lässt Richtungen erstarken, die die Demokratie gefährden können. Um der Orientierungslosigkeit vieler Menschen in der Schweiz entgegenzuwirken ist es wichtig, dass wir die Werte wieder entdecken, die Bestand haben und die unsere Gesellschaft tragen. Damit wir, unsere Kinder, Enkel und Urenkel in einer freien, friedlichen und sicheren Schweiz leben dürfen.»?

Viele Fragen tauchen auf. Was sind «schwache» Werte? Wie kann man Werte unterscheiden, die Bestand haben, von solchen, die das nicht tun? Durch unmittelbare Beobachtung oder - zwangsläufig - nachdenkliche

8 URL: www.zukunft-ch.ch, abgerufen am 19. 2. 2018

9 Ebd. 
Reflexion? Dann werden «vier Säulen schweizerischer Identität» genannt, die glücklicherweise keineswegs Alleinstellungsmerkmale schweizerischer Identität sind, sondern sich wesentlich breiterer Zustimmung über die Grenzen der Schweiz hinaus erfreuen: «Respektierung der Menschenrechte (Allgemeine Erklärung der Menschenrecht von 1948)», «Freiheit», «traditionelle [bzw.] natürliche Ehe und Familie», «Rechtsstaat» und «Würde des Menschen». ${ }^{10}$

Werte werden in diesem Zusammenhang auf zweierlei Weisen vorgestellt. Sie sind einerseits dauerhaft vorhanden. Dass wir sie «wieder entdecken», sei die anstehende Aufgabe. Andererseits gelte: «Der verbindliche Wertekonsens, der die Schweiz in den letzten Jahrhunderten getragen hat, ist zunehmend abgeschafft bzw. zerstört worden.» ${ }^{11}$ Welcher Konsens von Werten kann von Ferne den Anspruch erheben, über Jahrhunderte hinweg idealerweise angestrebt, geschweige denn verbindlich durchgesetzt worden zu sein? «Einst galten Vaterlandsliebe, Gehorsam, Demut, Opferbereitschaft oder Heldenmut unbestritten als ehrwürdige Schweizer Werte. «Heute liegen sie auf der Müllhalde, ohne dass noch irgendjemand Verwendung hätte für sie..»12

Vor allem aber fordert die Stiftung in ihrem Manifest selbst weitgehende Freiheiten: «Wir warnen vor jeder totalitären religiösen, ideologischen und philosophischen Tendenz, welche die Freiheit von Religion und Kultur behindert.»

Die vier Säulen stammten «aus dem biblischen Menschenbild», seien über Jahrhunderte hinweg entstanden. Dagegen ist einzuwenden, dass man den uneinholbaren Reichtum der biblischen Überlieferung unterschätzt, wenn man nur ein einziges und einheitliches Menschenbild er-

10 Ebd.

11 Ebd.

12 Thomas Ribi in der Leserdebatte der NZZ, Was sind überhaupt Schweizer Werte?, vom 1. 8. 2017, abgerufen am 19.2. 2018. hebt. Vielmehr ist in den biblischen Schriften viel mehr zu finden. In der Bibel wird u. a. eine ungefähr tausendjährige Geschichte des permanenten Wertewandels samt seiner anthropologischen und theologischen Vorannahmen dokumentiert, etwa das langsame Wachstum des Monotheismus, der Übergang von der Vielehe zur Einehe, die Durchsetzung der Geldwirtschaft neben dem Tauschhandel, der Übergang vom Nomandentum zur Sesshaftigkeit, die einsetzende Individualisierung und alle mit diesen Entwicklungen verbundenen Rückschläge, die Suche nach Kompromissen, das Scheitern und Gelingen.

Für die Diakonie sind Ausführungen zur Menschenwürde immer von besonderem Interesse. Die Stiftung stellt fest: «Jeder Mensch hat eine unantastbare Würde. Sie ist in seiner Gottebenbildlichkeit begründet und ein rechtlich geschütztes Gut. Der Einsatz für die Unantastbarkeit der Menschenwürde, auch für Ungeborene, Alte und Menschen mit Behinderungen ist deshalb grundlegend für den Fortbestand und die Kultur einer Gesellschaft». Thematisch unverbunden heisst es kurz zuvor freilich: «Natürliche Ehe und Familie: Sie bietet die besten Bedingungen für das Aufwachsen von gesunden und leistungsfähigen Männern und Frauen. Wir wehren uns gegen die gesellschaftliche Abwertung und rechtliche Auflösung der Ehe und Familie». Lohnt es nicht auch, sich gegen eine gesellschaftliche Überbewertung von Gesundheit und Leistungsfähigkeit zu wehren? Gibt es nicht nur «schwache», sondern dann auch «überstarke» Werte?

An erster Stelle steht für die Stiftung jedoch die Auseinandersetzung mit und die Angst vor dem «schariageleiteten Islam», dem ein Platz oberhalb gesellschaftlicher Wertdiskurse zugewiesen wird: «Der Islam und sein Rechtssystem, die Scharia, stehen über jedem nationalen und internationalen Recht.» Diese befremdliche, in islamistischen Kreisen zu vermutende Einordnung begegnet sogar mehrmals: «Man will nich wahrhaben, dass die Scharia über jedem nationalen und jedem internationalen Recht, auch über den Menschenrechten steht.» Das «StiftungsWir» will jedoch «eine schleichende Einführung der Scharia verhindern», denn «unsere Demokratie unterscheidet sich wesentlich von Rechtssys- 
temen aus anderen Kulturkreisen und ist für unsere Identität und den inneren Frieden unverzichtbar..» ${ }^{13}$

Dabei geht jedoch die geforderte Achtung der Menschenwürde durchaus hier und da vergessen. Prominent zitiert wird ein Zeitungsartikel von Eugen Sorg, in dem eingangs die Rede vom «Import von unzähligen Menschen aus gänzlich fremden Kulturen» ${ }^{14}$ ist. Für unzählige Schweizerinnen und Schweizer war es im 19. Jahrhundert ihrerseits überlebenswichtig, als Wirtschaftsflüchtlinge das Land zu verlassen und andernorts Zukunft und Leben zu suchen. Heutige, sogenannte Einheimische sollten aufgrund dieser historischen Prägung speziell sensibel sein, um Menschen nicht importierten oder exportierten Waren gleichzustellen.

Der besondere Fokus der Stiftung auf die Scharia lässt andere Probleme, die unsere Freiheiten bedrohen und einschränken, aus dem Blick geraten. Stellvertretend für viele andere seien nur weltweiter Klimawandel, nukleare Aufrüstung, Menschen- und Waffenhandel als Stichworte genannt.

Interessant ist schliesslich auch der kleine Wertewandel, der in wenigen Zeilen der Stiftung Zukunft $\mathrm{CH}$ zu beobachten ist. Während eingangs eine freie, friedliche und sichere Schweiz angestrebt wird, heisst es am Ende des Gedankenganges, dass «Freiheit, Frieden und Wohlstand» erhalten bleiben mögen. Sicherheit und Wohlstand müssen bei aller Freiheit in der Interpretation als zwei verschiedene Werte angesehen werden. Vor allem aber gehören Sicherheit und Wohlstand kaum zum inneren

13 Stiftung Zukunft, ebd (Anm. 8).

14 Eugen Sorg, Mohammeds Sieg - Der beliebteste Babyname und dessen Zusammenhang mit der europäischen Integrationspolitik, Basler Zeitung Online, 19. Januar 2018, zit. n. www.stiftung-ch.ch, abgerufen am 24. 2. 2018.
Kreis christlicher Werte, die prägend waren für die oben genannten vier Säulen.

Zusammenfassend wird deutlich, dass es nicht einfach möglich ist, ganz bestimmte Werte, nicht einmal in einem zuvor definierten Rahmen, wiederzuentdecken.

\section{Wertewandel? Eine Frage der Ehre}

Andreas Urs Sommer $(* 1972)$ hat einen wesentlichen und vielbeachteten Beitrag zum Gespräch über Werte in seinem Buch «Werte. Warum man sie braucht, obwohl es sie nicht gibts, ${ }^{15}$ geleistet.

Darin widerlegt er zunächst die Annahme, Werte seien immer schon dagewesen, nur sehr spät und von zu wenigen wieder entdeckt worden, so dass man sie nur allgemeiner und erneut bekannt machen müsste. Vor allem gibt es keinen Wertzusammenhang, der unwandelbar gleich galt und gilt. Um es an der vielbeschworenen Freiheit zu illustrieren:

«Der Banker versteht unter Freiheit etwas anderes als der Häftling, der Sozialrevolutionär etwas anderes als der religiös Erweckte. Und doch lassen sie sich alle auf Freiheit einschwören, wobei sie aus dem angeblichen An-sich-Wert «Freiheit ganz unterschiedliche Handlungs- und Denkfolgerungen ziehen.» ${ }^{16}$

Besondere Aufmerksamkeit widmet Sommer sodann den sogenannten christlichen Werten, die es als solche noch gar nicht lange gibt. Bezeichnenderweise wurden sie zuerst bei Friedrich Nietzsche so genannt, kei-

15 Stuttgart 2016. Der Autor lehrt Philosophie an der Universität Freiburg i. B. und leitet die Forschungsstelle Nietzsche-Kommentar der Heidelberger Akademie der Wissenschaften. Der Titel des Buches stammt nicht von ihm, sondern von Franziska Remeika, vgl. ebd., 176

16 Ebd., 18 
neswegs in lobender Absicht. Als umzuwertende, christliche Werte bezeichnete Nietzsche u. a. Selbstherabsetzung, Selbsterniedrigung und eine oft missbrauchte Idee von Demut.

«Das war Christen bis dahin nie eingefallen, die stattdessen «christliche Werke» propagiert hatten. «Christliche Wertes hätten unter Christen erst im 20. Jahrhundert Kredi gewonnen, parallel zum Niedergang des christlichen Glaubens. Denn unter «christlichen Werten könne jeder sich vorstellen, was er wolle..) ${ }^{17}$

Erst im 20. Jahrhundert traten dann die sogenannten christlichen Werte ihren sprachlichen Siegeszug unter Christinnen und Christen an.

Anzumerken ist, dass es Sommer seinerseits nicht gelingt, die Entwicklungen des Christentums einigermassen wertneutral zu beschreiben. Eine teilweise Unkenntnis und ein wohlfeiles Vorurteil gehen eine unerquickliche Allianz ein, wenn er schreibt: «Das Christentum hat den Wert des Leidens in der Nachfolge des leidenden Erlösers großgeschrieben, so lange dieses Christentum noch eine ernstzunehmende Alternative zur Diesseitsbejahung dargestellt und sich noch nicht in Wohlfühl- und Nettigkeitsplattitüden aufgelöst hat.» ${ }^{18}$ Ein oberflächlicher Blick auf die diakonischen Herausforderungen, $z$. B. in der Mitte des Lebens im Umgang mit einer menschenunfreundlichen Arbeitswelt oder am Lebensende im Umgang mit einem ökonomiestrategisch organisierten Gesundheitssystem, könnte ihn vom Gegenteil überzeugen.

Der Wertewandel, vor allem aber der Werthierarchienwandel soll an einem exemplarischen Wert nachgezeichnet werden, der einerseits einst

17 Ebd., 35f. Die Einsicht, dass die Rede über «christliche Wertes erst mit und kirchlich nach Nietzsche begann, stammt nicht von Sommer, sie gehört zu den Perspektiven christlicher Ethik, vgl. z. B. Martin Honecker, Evangelische Ethik als Ethik der Unterscheidung. Mit einer Gesamtbibliographie von Martin Honecker, Münster 2010, 158.

18 Sommer, Werte (Anm. 15), 150. als prägender christlicher Wert figurierte, andererseits die aktuelle diakonische Arbeit mit Flüchtlingen unterschiedlichster Kulturkreise und religiöser Einstellungen vor neue Probleme stellt.

Es geht um die Frage der Ehre. Vor ungefähr 150 Jahren waren die Ehre und die Konsequenzen bei ihrer auch nur vermuteten oder geringfügigen Verletzung massgeblich für die Beurteilung und Aufrechterhaltung bzw. für das dramatische Ende von Ehen. Ethische Grundlagenwerke von christlichen Theologen befassten sich seinerzeit ausführlich mit diesen Fragen; lösungsorientiert ging es um Pro oder Contra zum Duell.

Friedrich Heinrich Christian Schwarz (1766-1837), Pädagoge und Theologe, bringt in seiner «Evangelisch-christliche[n] Ethik: Handbuch für Theologen und andere gebildete Christen» aus dem Jahre 1830 zahlreiche Argumente gegen das (zu) weit verbreitete Duell vor. Das ausführliche Zitat zeigt, dass damals gerade traditionelle Werte unter schön scheinenden Namen und ihre dazugehörigen Sitten negative Konsequenzen hatten. Weder die allgemeine Akzeptanz noch das Alter der Gebräuche sind nach Schwarz Gründe gegen einen tiefgreifenden Wertewandel, den er mit seiner Schrift befördern möchte - im Namen christlicher Lehre.

«Eine beklagenswerthe Sitte, die man mit dem schönen Namen Ehrensache zu kleiden pflegt, findet wohl hier den schicklichsten Ort ihrer Beurtheilung - das Duell. Das Christenthum hat entschieden und schon längst solchen Zweikampf um der Ehre willen als Unsitte verworfen, weil es über jeden Selbstmord den Stab bricht. Denn das Duell ist und bleibt, wo nicht ein doppelter wirklicher Selbstmord, wenigstens ein zweifaches Attentat verbrecherischer Menschentödtung. [...] Begreiflich genug ist es, dass das Vorurtheil, worin die Duelle so fest halten, tief eingewurzelt ist, denn im gemeinen Leben verwechselt man fast durchaus die Würde mit der Ehre, insbesondere bei der Kriegs- und Ritterehre, und wie mächtig zwingt nicht die Gewalt herge- 
brachter Nationalsitte! Es gibt vielleicht keinen stärkeren psychologischen Zwang, als der ist, wodurch sich Mancher zum Duell genöthigt hält, ein wahrer Nothstand, der den Gesetzgebungen keine Ruhe läßt, bis sie ihm abgeholfen haben.» ${ }^{19}$

Wie stark eine vermutete und als besonders alt geltende «Nationalsitte» sich nach wie vor auswirken kann, ist u. a. oben am dargelegten Beispiel der vier Säulen schweizerischer Identität deutlich geworden. Wie weit Schwarz seiner Zeit voraus war und wie wenig Einfluss er offenbar mit seinem ethischen Handbuch nehmen konnte, lässt sich daran ermessen, dass die Frage der Ehre und die Konsequenzen eines Duells wenig später einen berühmten Roman wert waren.

Der Schriftsteller Theodor Fontane (1819-1898) gilt als literarischer Spiegel Preussens und als bedeutendster deutscher Vertreter des Realismus. Er verfasste 1894/95 einen Roman zu Eheehrproblemen, «Effi Briest» ${ }^{20}$, der knapp einhundert Jahre später immer noch zur Schullektüre, sogar in der DDR, gehörte. Die Protagonistin oder genauer Hauptleidtragende wird als 17jährige auf Zureden ihrer Mutter mit dem mehr als doppelt so alten Baron von Instetten verheiratet. Er behandelt seine junge Ehefrau wie ein Kind und vernachlässigt sie stark. Effi hat ihrerseits Zeit, Langeweile und eine vorübergehende Liebschaft mit einem wenig vertrauenerweckenden, aber wilden Offizier. Einige Jahre später findet Effis Ehemann durch einen unglücklichen Zufall alte Liebesbriefe der beiden und tötet - ausdrücklich aus gesellschaftlich gefragten Gründen der Ehre, gegen seinen eigenen, empfundenen und erklärten Willen - den Offizier im Duell.

19 Friedrich Heinrich Christian Schwarz, Evangelisch-christliche Ethik. Handbuch für Theologen und andere gebildete Christen, Heidelberg 1830, 187.

20 URL (Quelle mit Suchfunktion): gutenberg.spiegel.de/buch/effi-briest-4446/1, abgerufen am 23. 2. 2018. Alle weiteren Zitate aus dem Roman ebd.
Er ruiniert damit wissentlich drei Menschenleben. Der Offizier ist sofort tot, Effi stirbt drei Jahre lang, er selbst bleibt lebenslänglich gestraft mit seiner Tat. Bereits Schwarz hatte den äusserst starken psychologischen Zwang exakt beschrieben, durch den sich mancher gegen sein Gewissen und gegen seinen Willen zum Duell gezwungen sieht.

Effi resümiert mit der Schärfe ihres Verstandes: «Ehre, Ehre, Ehre [...] und dann hat er den armen Kerl totgeschossen, den ich nicht einmal liebte und den ich vergessen hatte, weil ich ihn nicht liebte. Dummheit war alles, und nun Blut und Mord.» Effis letzter Wille bezieht sich nochmals auf einer anderen Ebene auf die Ehre: «Ich möchte auf meinem Stein meinen alten Namen wiederhaben; ich habe dem andern keine Ehre gemacht.»

Baron von Instetten spricht vor dem Duell gegenüber seinem Freund vom «Fleck auf meiner Ehre» - «jenes [...] uns tyrannisierende Gesellschafts-Etwas, das fragt nicht nach Scharm [sic!] und nicht nach Liebe und nicht nach Verjährung. Ich habe keine Wahl, ich muss.» Freund Wüllersdorf antwortet darauf: «Das mit dem «Gottesgericht», wie manche hochtrabend versichern, ist freilich Unsinn, nichts davon, umgekehrt, unser Ehrenkultus ist ein Götzendienst, aber wir müssen uns ihm unterwerfen, solange der Götze gilt.» Wie weit Schwarz seiner Zeit voraus war und wie hartnäckig sich der Götze Ehre trotz Fontanes Meisterwerk am Leben hält, wird noch kurz vor Ende des 20. Jahrhunderts deutlich.

Wolfgang Trillhaas musste in seinem Lehrbuch der Ethik Fontanes Überlegungen nur leicht aktualisieren.

«Aber die Reizbarkeit des Ehrgefühls und die Überbetonung des point d'honneur, wie sie nicht nur in gewissen Ländern und in früheren Zeiten kultiviert wurde, sondern auch gewisse Schichten der Bourgeoisie bis in die Gegenwart hinein kennzeichnet, entstellt hier doch die wahren Sachverhalte. Die Ehre wird nämlich nun zu einem Privileg des vornehmen Mannes. Und zu diesen Privilegien wird es gerechnet, dass der Vornehme seine verletzte Ehre nicht nur wiederherstellen muss, sondern dass er sie unter Umgehung der öffentlichen Gerichte durch einen Beweis des Mutes wiederherstellen muss. Mit der Überempfindlichkeit des Ehrgefühls geht seit alters Hand in Hand eine gewisse Mythologie und ein verhängnisvolles Pathos. Die be- 
fleckte Ehre ist nur mit «Blut zu reinigen, und der Beleidigte ist zur Rache an seinem Beleidiger nicht nur berechtigt, sondern geradezu verpflichtet..) ${ }^{21}$

Diese Vorstellungenskonglomerate, so überholt sie uns erscheinen mögen, so freudig wir den erfolgreichen Wertewandel, ja den Werthierarchienwandel seit dem 19. Jahrhundert begrüssen mögen, erscheinen erneut und stehen aktuell zur Diskussion, wenn es etwa um Zwangsheiraten, Ehrenmorde und Fragen der Ehre in Grossfamilien fern und besonders nah geht, die oftmals, jedoch nicht immer mit einem bestimmten Verständnis des Islam einhergehen.

Ahmad Mansour ${ }^{22}$ engagiert sich seit Jahren als Gruppenleiter des Berliner Antigewalt-Projekts HEROES, das sich aktiv gegen jede «Unterdrückung im Namen der Ehre» wendet. Denn ein «traditioneller Ehrbegriff, Gehorsam und Angst prägen in vielen Migrantenfamilien die Erziehung. Die 〈Heroes〉 wollen Jugendliche überzeugen, unabhängiger zu werden.» ${ }^{23}$ Dazu gibt es viele gute Gründe, die deutlich werden, wenn man sich die Diskussion in einer gegenwärtigen Berliner Schulklasse vergegenwärtigt, die Mansour genau dokumentiert hat.

21 Wolfgang Trillhaas, Ethik, Berlin/New York 31986, 359 mit einem Exkurs zum Duell und Zweikampfsitten insgesamt.

22 Ahmad Mansour, geboren 1976, ist arabischer Israeli und lebt seit 2004 in Berlin. Er ist Diplom-Psychologe und arbeitet für Projekte gegen Extremismus, u.a. bei HEROES, einem Projekt gegen Unterdrückung im Namen der Ehre und für Gleichberechtigung, und bei HAYAT, einer Beratungsstelle für Deradikalisierung. Außerdem ist er Programme Director bei der European Foundation for Democracy. Für seine Arbeit erhielt er den Moses-Mendelssohn-Preis zur Förderung der Toleranz. Er hat zahlreiche Veröffentlichungen zum Thema Salafismus und Antisemitismus vorgelegt. URL: www.fischerverlage.de, abgerufen am 24. 2. 2018.

23 Ahmad Mansour, Ehre und Familie. «Wenn mein Bruder mich schlägt, härtet mich das ab», in: DIE ZEIT ONLINE vom 27. 2. 2013, abgerufen am 23. 2. 2018. Die weiteren Zitate ebd.
Beim Studium der Schülervoten fällt zunächst die grosse Diversität der nebeneinander existierenden muslimischen Überzeugungen und Haltungen auf. Mansour berichtet aus einem Workshop einer 9. Klasse in Berlin zum Thema Ehre.

«Sema ist 15, mitten in der Pubertät, auf der Suche nach sich und ihrem Lebensentwurf. Nur zum Thema Ehe hat sie bereits feste Ansichten. «Mein Mann darf mich schlagen, wenn ich einen Fehler mache, sagt sie. Verheiratet ist sie freilich noch nicht, nein, sie spricht von einer vorbestimmten Zukunft. Lachend fügt sie hinzu: «Und wenn mein Bruder mich schlägt, dann härtet mich das ab.» Ihr Lachen klingt gepresst. Diese Gewalt geschieht ihr jetzt. Dicker, schwarzer Lidstrich und viel Wimperntusche betonen Semas ausdrucksstarke Augen. Sie trägt einen langen Pullover, enge Jeans, High Heels. Wie viele ihrer Klassenkameraden ist Sema Muslima. Als solche will sie in der Gruppe auch geachtet werden.»

Mansour führt diesen Workshop nicht allein, sondern mit der Organisation Heroes durch. Diese speziellen Helden sind Jugendliche und junge Erwachsene, die ihrerseits mit problematischen Vorstellungen von Ehre gross geworden sind, Semas Interpretation aktueller, familiärer Gewalt und ihre Zukunfts- und Eheaussichten aus ihren eigenen Herkunftsfamilien und Biographien kennen und in der Lage sind, sie kritisch zu hinterfragen. Mansour ist überzeugt, dass nur von innen heraus ein Kulturwandel, eine Reform von Werthierarchien, möglich ist. Wie weit der Weg ist, wird an Fatihs Diskussionsbeiträgen deutlich:

«Der gleichaltrige Fatih pflichtet ihr bei: \Die Ehre eines Mannes», sagt er cool, «steckt zwischen den Beinen einer Frau.» Damit zitiert er ein türkisches Sprichwort. «Meine Schwester muss Jungfrau bleiben’, erläutert Fatih. Sie dürfe keinen Freund haben, sauch nicht auf Facebooks. Und am Abend soll sie sich nicht draußen herumtreiben. 'Wenn sie es doch tut, muss ich sie schlagen.» In der Klasse wird Widerspruch laut: «Bin ich froh, dass ich nicht deine Schwester bin`, sagt ein Mädchen.»

Es fällt nicht allzu schwer, einen angeregten Austausch zum Thema Ehre zwischen den fast Gleichaltrigen Effi und Sema und ein Gespräch unter Männern zwischen Baron von Instetten und Fatih zu imaginieren, auch 
wenn der Graben von fast zwei Jahrhunderten, zwei Religionen und zwei Kulturen zu überbrücken ist. Ohne den Abstand der Jahrhunderte wären sie sich jedenfalls alle in Berlin begegnet. Mansour charakterisiert Fatih und Sema im Kontext ihrer Familien, weil diese das wirksamste Wertsystem darstellen, trotz aller Einflüsse durch Klassenkameraden oder Massenmedien.

«Wie Sema gehört Fatih zur sogenannten dritten Generation von Immigranten in Deutschland. Seine Großeltern sind vor 40 Jahren aus Anatolien hierher zum Arbeiten gekommen. Fatih lebt mit seinen drei Geschwistern und den Eltern in einer VierZimmerwohnung in Nord-Neukölln. Er ist Deutscher. Aber seine Ehre? Die stammt aus einer anderen Welt.»

Es ist zwar weit von der anderen Welt in die eine Welt, jedoch zugleich näher als man zu denken gewöhnt ist. Kulturunterscheidende Arroganz ist auf jeden Fall fehl am Platz. Das alte Preussen und die Türkei sind sich näher als die Landkarte denken lässt; in Berlin sind das Preussen zu Fontanes Zeiten leicht zu vergegenwärtigen und die aktuellen Fragen der Ehre in emotionaler Auseinandersetzung unmittelbar anzutreffen:

«Einer sagt, was er vermutlich von Vater oder Onkel hört: Lieber fünf kriminelle Söhne als eine verhurte Tochter.` Ein anderer behauptet: \Ich würde meine Schwester umbringen, wenn sie Sex vor der Ehe hat.) Dort, wo diese Jugendlichen groß werden, gelten Werte wie Selbstbestimmung, Emanzipation der Frauen und Individualität als Auflehnung gegen die Familie. Die Eltern kennen es nicht anders. Ihr Ziel ist es, die Familie in der Fremde zusammenzuhalten. Sie fühlen sich sicherer, wenn die Hierarchien so klar und eng sind, wie sie es von den eigenen Müttern und Vätern kennen. Alle müssen den Älteren gehorchen. Männer stehen über Frauen, Brüder über Schwestern. Von klein auf hören die Kinder, dies sei die eiserne Ordnung einer Welt, die Allah gefällt.

Die Fragen der Ehre sind also alt-aktuell und die Beispiele alt-aktueller Werte liessen sich vermehren. Weil das so ist, müssen Werte kommuniziert und bewertet werden.

\section{Wertschätzung? Eine Frage der Kommunikation}

Kein Wert steht unabänderlich fest. Kein Wert versteht sich von selbst, obwohl Werte immer dann ins Spiel gebracht werden, wenn fraglos und unstrittig festgestellt werden soll, was anzustreben sei. Aber Werte haben ein reiches und wechselvolles Innenleben. Sowohl zur Zeit Fontanes als auch in den Communities von Sema und Fatih war und ist, um beim eingeführten Beispiel zu bleiben, Ehre ein äusserst komplexer Begriff.

Ahmet Toprak ${ }^{24}$ nennt die vier Komponenten des traditionellen, türkischen Ehrbegriffes: Ansehen, Schutz der Schwächeren, Respekt und Würde. ${ }^{25}$ Obwohl also Ehre in den Grundsätzen der Stiftung Zukunft $\mathrm{CH}$ nicht als Begriff begegnet, geht es auch dort explizit um Schutz, Respekt und Würde, implizit auch um das Ansehen von Menschen. Freilich unterscheiden sich trotz der Themenähnlichkeit Kontext und Kultur und damit verbundene, jeweilige Freund- und Feindbilder gravierend voneinander.

Ahmet Toprak konfrontiert danach das vierteilige Idealbild der Ehre mit der Realität: «In den Aussagen der Jungs [...] wird 〈Ehre» auf die Sexualmoral der Frau reduziert. Das ist der Knackpunkt, der mich immer wütend macht. Denn Ehre ist viel mehr.» ${ }^{26}$ Toprak könnte von ungewohnter Seite Zustimmung erhalten. Auch Fontane klagt mit seinem Roman «Effi Briest» die Reduktion der Ehre auf die Sexualmoral an. Dass mit der Reduktion gleichzeitig eine Vergötzung verbunden sein kann, überbrückt einerseits die sonst grosse Kluft zwischen den Kultu-

24 Ahmet Toprak $(* 1970)$ ist Professor für Erziehungswissenschaften an der Fachhochschule in Dortmund. Er ist Deutscher mit türkischer Herkunft.

25 Diskussionsbeitrag von Ahmet Toprak, in: Ehre ist das wichtioste. Religionen im Gespräch 7/2013 im Haus der Religionen in Hannover am 18. 4. 2013 unter Moderation von Prof. Dr. Wolfgang Reinbold, 2.

26 Toprak, Ehre (Anm. 25). 
ren, macht aber andererseits die Probleme nicht kleiner. Die Komplexität der Wertdebatte zwischen den Kulturen und Religionen ist damit skizziert. Ehre als möglicher Deckmantel für Angst und Gehorsam, als Machtfaktor und identitätsstiftendes Merkmal gerade oder gar nur dort, wo man sich ausgegrenzt fühlt, kommen ausserdem dazu. ${ }^{27}$

Neben dem reichen Innenleben sind Werte darüberhinaus sehr lebendig. Sie können sich sogar vermehren. Andreas Urs Sommer nennt «Achtsamkeit» und «Empathie» als neuesten Nachwuchs bei den Werten und beobachtet: «Neue Werte scheinen oft situativ leistungsfähiger als das etablierte Werteset. ${ }^{28}$

Aber gibt es überhaupt etablierte Wertesets? Sommer glaubt zu wissen, wo sie wohnen. Er vermutet sie innerhalb von «Kirchen und sonstiger Bekenntnissondergruppen» ${ }^{29}$, wie er sich, selbst wenig um den Wert Wertneutralität bekümmert, ausdrückt.

Fragt man aber genau nach, d.h. fragt man nach wesentlichen Werten in - relativ zur pluralen Gesellschaft - homogenen, christlichen Gruppen, etwa unter reformierten Pfarrerinnen und Pfarrern eines Dekanates oder unter Sozialdiakoninnen und Sozialdiakonen oder an einem Frauenmorgentreff, wird man der Fülle möglicher Wertsetzungen ansichtig, nicht jedoch einer übersichtlichen, geschweige denn annähernd einheitlichen Orientierung. Weder innerhalb einer Generation, noch am selben Lebensort oder in derselben Kirchgemeinde, noch unter Berufsleuten lässt sich ein «etabliertes Werteset» finden, das diesen Namen verdient. ${ }^{30}$

27 Vgl. die Wortbeiträge Mansour im zitierten Gespräch der Religionen (Anm. 25), 3 und 12.

28 Sommer, Werte (Anm. 15), 56.

29 Ebd., 76.

30 Hier gebe ich meine Erfahrungen an Vorträgen und Workshops in reformierten Landeskirchen zum Thema Werte wieder.
Selbst dann, wenn man in Workshops an den genannten Orten einen Wertekanon von 30 bis 40 Werten vorgibt, ändert sich nichts an der Vielfalt des Resultats. Zunächst wird ein Dutzend Werte eigenständig ergänzt, deren Fehlen in der grossen Menge des Gegebenen dennoch auffällt. Sodann konnte ich beobachten, dass selbst in kleinen Arbeitsgruppen teilweise aufwändige demokratische Verfahren entwickelt und erprobt werden, um die sehr weit auseinanderliegenden Wertvorstellungen von wechselnden Mehr- und Minderheiten angemessen zur Geltung, in eine Rangfolge und miteinander ins Gespräch zu bringen. Christliche Werte, wenn man sich denn auf diese Begrifflichkeit einlassen will, gib es nur als disparate (Über)fülle, sie stellen keinen Kanon dar, den man nur wiederfinden und studieren müsste.

Mit dieser Wert(über)fülle sind Christinnen und Christen nicht allein. Die plurale Situation der Wertwahrnehmungen dokumentiert auch Ahmad Mansour in der Berliner Schulklasse unter den muslimischen Schülerinnen und Schülern. Bei weitem nicht alle denken, glauben und leben wie Sema und Fatih.

«Glücklicherweise finden sich in den Klassen auch immer wieder Jungen wie Mädchen mit Migrationshintergrund, die solche Konzepte der Unterdrückung ablehnen. Zwar kennen fast alle ähnliche Vorstellungen - aber in verschiedener Intensität Manche haben Lehrer oder Nachbarn, die sie beeinflusst haben, viele haben Eltern, die liberaler denken. Fast alle kennen die hiesigen Medien, erfahren in Serien, Comedy-Shows mit Bülent Ceylan, Kaya Yanar oder Django Asül, dass man spielerischer mit Stereotypien umgehen kann.»31

Eine Aufgabe besteht also darin, schon unter Berufskollegen und in Schulklassen die unausweichliche Vielfalt der Werte im kleinen Kreis

31 Mansour, Ehre (Anm. 25). 
bewusst zu machen, auszuhalten und in einem «Dialog zur Erkundung des Guten» ${ }^{32}$ weiterzuentwickeln.

Wie so ein einzelner Wertweiterentwicklungsschritt aussehen kann, beschreibt Ahmad Mansour. Die damit verbundenen Mühen und Hoffnungen treten deutlich zutage.

«In einem Sketch spielen die Heroes vor, wie ein muslimischer Vater seine Tochter dabei unterstützt, Abitur zu machen und alles gibt, damit sie sich später beruflich verwirklichen kann. Er tut das gegen den Widerstand der Familie, gegen den Rat sei nes ältesten Bruders. Sema und ihre Klassenkameradinnen staunen. Vorsichtig sprechen sie von Träumen, in denen das Wort «Unabhängigkeit vorkommt [...].

Finanziell unabhängige Ehefrauen, so dämmert den Jungen, müssen sich nicht alles gefallen lassen. Sie sind nicht vom Mann abhängig, sie könnten ihn abweisen oder sogar verlassen. Es braucht Zeit und Geduld, bis sie bereit sind, Strukturen von Kontrolle und Macht in Frage zu stellen. [...]

Und Fatih? Ihm ist bewusst geworden, dass seine Mutter ihm nicht bei den Hausaufgaben helfen kann, weil sie kaum lesen kann. Dass es schön wäre, wenn sie sich für seine Schule interessieren könnte. Er, der im Workshop noch erbittert auf die 〈Tradition> gepocht hatte, sagt hinterher: (Toll, das war voll krass. Macht weiter Jungs!») 33

Theodor Fontane, Ahmad Mansour und Ahmet Toprak verbindet ihr Interesse, den Wert «Ehre» nicht zu lassen, was er einmal war und wofür er gegenwärtig gehalten wird. Sie sind einig, dass an einer neuen Werthierarchie und einem neuen Wertverständnis aktiv gearbeitet werden muss Fontane tut das mit seinem Roman, Mansour mit Schulworkshops, Toprak in der Ausbildung von Pädagoginnen und Pädagogen. Alle dre missbrauchen dabei auch nicht den Wertbegriff «Toleranz» als Tarnname

32 Jürgen Becker, Zugänge zur Bergpredigt, in: Kieler Entwürfe für Schule und Kirche 15 (1994), 3-20 (19)

33 Mansour, Ehre (Anm. 25). für Gleichgültigkeit und Desinteresse, wie es allzu oft geschieht, sondern setzen sich den Konflikten ihrer Zeit aus.

Nicht zu unterschätzen ist in diesen Zusammenhängen die offenbar kulturübergreifende Dauerbeobachtung, unter der alle Menschen stehen, die bestimmte Werte realisieren oder gerade nicht. Fontane beobachtet im pommerschen Dorf, «es sei doch zuviel verlangt, bloß um des Geredes der Kessiner willen auf etwas zu verzichten, das einem so viel wert sei.»34

Mansour kennt es aus der orientalischen Kultur und hat einen genialen Begriff für die kollektive Dauerbeobachtung des Individuums: «Üblicherweise hat «Ehre` viel zu tun mit dem Dorfauge. [...] Das Dorfauge kontrolliert alles und schaut, wie jemand sich verhält, wie die Frauen der Familie sich verhalten.» ${ }^{35}$

Werte, ganz gleich, woher sie stammen und wie und wo sie in Geltung stehen und beobachtet werden, sind auf Kommunikation angewiesen. «Wir müssen offen über die Probleme reden. Wir dürfen nicht verharmlosen. Wir dürfen die Probleme nicht in einem Kulturrelativismus verstecken oder einfach schweigen, wie es in vielen Städten passiert... ${ }^{36}$ Kommunikation über Werte ist wertvoll, aber niemals unverfänglich und nebenwirkungsfrei. Schon Eberhard Jüngel warnte seinerzeit mit Bezug auf Carl Schmitt vor dem Konfliktpotential von Wertdiskussionen, wenngleich das damals gewählte Beispiel völlig unspektakulär zu sein scheint.

«Wertdenken ist eminent aggressiv. [...] Das zeigt sich gerade, wenn Werte aufeinanderstoßen oder auch nur unterschiedliche 〈Realisierungen〉 derselben Werte (z. B. der-

34 Theodor Fontane, Effi Briest, 16. Kapitel, URL: gutenberg.spiegel.de/buch/effibriest-4446/16, abgerufen am 25. 2. 2018

35 Wortbeitrag Mansour, in: Religionen im Gespräch (Anm. 25), 12

36 Ebd. 
selben «Grundwertes in CDU und SPD) miteinander konkurrieren. Diese simmanente Aggressivität, die «mit der thetisch-setzerischen Struktur des Wertes von selbst gegeben ist und «durch den konkreten Vollzug des Wertes immer von neuem produziert wird, bbleibt die fatale Kehrseite der Werte..» ${ }^{37}$

Um dieser Gefahren willen aber die kritische Kommunikation über Werte einzustellen, wäre fatal. Auch die (Über)fülle von Wertsetzungen entbindet nicht von der Pflicht, sich mit ihnen auseinanderzusetzen. Sommer schlussfolgert:

«Werte sind Projektionsflächen, die es jeder und jedem erlauben, darauf einzutragen, was sie für konform mit ihren Bedürfnissen, Interessen, Präferenzen halten. Solche Projektionsflächen sind jetzt und künftig nötig, weil unterschiedlichste Menschen in modernen Gesellschaften zusammenfinden müssen, deren Bedürfnisse, Interessen, Präferenzen denkbar verschieden sind.» ${ }^{38}$

Für die Diakonie spielen dabei nicht nur Werte in offener Konkurrenz eine zentrale Rolle, sondern auch alle jene Wertsetzungen, die zusammengehören, gerade weil sie so verschieden sind. So wie auch Puzzleteile nur dann genau zusammenpassen, weil sie nicht exakt gleich gestaltet sind

Im diakonischen Alltag, z. B. im Zusammenleben und Zusammenlernen mit Menschen mit Demenz oder Behinderungen, aber nicht nur dort, geht es immer um die Balance zwischen den Werten Fürsorge und Eigenverantwortung, die nicht von vornherein gegeben ist, sondern über die immer wieder neu entschieden werden muss. Weitere Beispielpaare sind leicht zu bilden: Freiheit und Verbindlichkeit, individuelle Emanzi-

37 Jüngel, Wahrheit (Anm. 2), 99 mit Zitaten von Carl Schmitt, Die Tyrannei der Werte, in: ders./Eberhard Jüngel/Sepp Schelz, Die Tyrannei der Werte, hg. v. Sepp Schelz, Hannover 1979, 9-43 (34). Das Werk erschien wegen bleibender Aktualität in 3., korrigierter Auflage in Berlin 2016.

38 Sommer, Werte (Anm. 15), $173 \mathrm{f}$ pation und ein Sinn für förderliche Gemeinschaften. Alles das sind Paare in einem Wertepuzzle, das durch dauerhafte Kommunikation stets neu zusammengestellt werden muss, niemals ein fertiges Gesamtbild ergeben wird, immer an fehlenden Teilen leiden wird, aber nach Zerstörungen und Zerfall auch immer wieder neu zusammengesetzt werden kann. Aber die Werte dafür sind reichlich vorhanden, wenn wir sie brauchen.

Autorin:

Dörte Gebhard,

Dr. theol., Pfarrerin in der reformierten Kirchgemeinde Schöftland, Privatdozentin für Praktische Theologie an der Theologischen Fakultät der Universität Zürich, Familienfrau 